Comment. Math. Helv. 79 (2004) 582-604

0010-2571/04/030582-23

DOI $10.1007 / \mathrm{s} 00014-004-0808-y$
(C) 2004 Birkhäuser Verlag, Basel

Commentarii Mathematici Helvetici

\title{
Combinatorics of rational singularities
}

Lê Dũng Tráng and Meral Tosun

\begin{abstract}
A normal surface singularity is rational if and only if the dual intersection graph of a desingularization satisfies some combinatorial properties. In fact, the graphs defined in this way are trees. In this paper we give geometric features of these trees. In particular, we prove that the number of vertices of valency $\geq 3$ in the dual intersection tree of the minimal desingularization of a rational singularity of multiplicity $m \geq 3$ is at most $m-2$.
\end{abstract}

Mathematics Subject Classification (2000). 32S25, 32S45, 15Q10, 05 C05.

Keywords. Classification of rational singularities, topology of singularities, dual graph, glueing of rational trees.

\section{Introduction}

Rational surface singularities are the singularities of normal surfaces whose geometric genus does not change by a desingularization. These singularities were studied for the first time by Du Val in [3].

Following the works of Artin [1], Spivakovsky (see [15], p. 421) has emphasized the fact that a complex normal surface singularity is rational if and only if the dual intersection graph associated with a desingularization of the singularity satisfies some combinatorial properties.

The dual intersection graph determines by plumbing the topology of the corresponding singularity. Conversely, Neumann proved in [13] that the dual intersection graph associated with the minimal good desingularization of a normal surface singularity (algebraic or analytic) is determined by the topology of the surface in a neighbourhood of the singularity. So, to obtain a topological classification of rational singularities of complex surfaces, it is important to study the graphs which are the dual intersection graphs associated with a desingularization of these singularities.

In this work, we study the graphs which satisfy the combinatorial conditions that characterize dual intersection graphs associated with desingularizations of rational singularities. Since these graphs are trees, we call them rational trees. We give several properties of these trees. In particular, we bound their complexity 
by means of an invariant of the tree which is interpreted as the multiplicity of an associated rational singularity. In this case, we measure the complexity of a graph by the number of vertices of valency $\geq 3$. The properties given in this paper also lead us to a complete list of the dual intersection graphs associated with the minimal desingularizations of rational singularities of multiplicity 5 (see [18]). The lists for the cases of multiplicity 2,3 , and 4 were already given in [3], [1] and [16] respectively.

\section{Rational singularities}

In this paragraph, we recall basic properties of rational surface singularities.

A surface singularity is a point $x$ on a complex analytic space $X$ whose local ring $\mathcal{O}_{X, x}$ has Krull dimension 2. The surface singularity is irreducible if the local $\operatorname{ring} \mathcal{O}_{X, x}$ is an integral domain. If $x \in X$ is an irreducible surface singularity, there is a neighbourhood of $x$ in $X$ where all the local rings have dimension 2 . If, furthermore, the local ring $\mathcal{O}_{X, x}$ is normal, i.e. an integral domain and integrally closed in its field of fractions, there is a neighbourhood $U$ of $x$ in $X$ where, for all the points $y$ in $U-\{x\}$, the local ring $\mathcal{O}_{X, y}$ is regular of dimension 2, i.e. isomorphic to $\mathbb{C}\{u, v\}$. In this case, we shall say that $(X, x)$ is a normal surface singularity.

Now, let $(X, x)$ be a normal surface singularity. We call desingularization of $(X, x)$ a proper analytic map $\pi: \tilde{X} \rightarrow U$ of a non-singular analytic space of dimension 2, i.e. a non-singular surface, onto a neighbourhood $U$ of $x$ in $X$, such that $U-\{x\}$ is non singular and:

i) the map $\pi$ induces an analytic isomorphism of $\tilde{X}-\pi^{-1}(x)$ onto $U-\{x\}$;

ii) the inverse image $\pi^{-1}(U-\{x\})$ is dense in $\tilde{X}$.

For more details, see [8].

Zariski's Main Theorem says that, when $\pi$ is not an isomorphism, the exceptional divisor $\pi^{-1}(x):=E$ is connected and has dimension 1 (see [6], Theorem V.5.2). A desingularization is called good if the divisor $E$ has normal crossings and each of its irreducible components is smooth and, it is called very good if, in addition, two distinct components of $E$ intersect transversally at most in one point. A desingularization might not be good, since the irreducible components of $E$ can be singular and intersect each other not transversally. However, by blowing up points, one can obtain from any desingularization a very good desingularization.

A positive cycle with support on $E$ is a formal sum of the irreducible components $E_{i}$ of $E$ with non-negative integral coefficients and with at least one positive coefficient. The set of positive cycles is naturally ordered by the product order. So, the positive cycle $\sum_{i} a_{i} E_{i}$ is bigger than $\sum_{i} b_{i} E_{i}$ if and only if $a_{i} \geq b_{i}$ for all $i$. The support of a positive cycle $\sum_{i} a_{i} E_{i}$ is the union of the components $E_{i}$ for which $a_{i} \neq 0$. The intersection number $\left(E_{i} \cdot E_{j}\right)$ of components $E_{i}$ and $E_{j}$ on $\tilde{X}$ is defined as the sum of the intersection numbers at the intersection points 
of $E_{i}$ and $E_{j}$, if $i \neq j$, which is a non-negative number, and the self-intersection number of $E_{i}$, if $i=j$, which is a negative number.

We associate a graph with the exceptional divisor $E$ of a desingularization as follows: To each component $E_{i}$ of $E$ we associate a vertex. If $i \neq j$ we link the vertices associated to $E_{i}$ and $E_{j}$ by $\left(E_{i} \cdot E_{j}\right)$ edges. We endow each vertex with the weight $-\left(E_{i} \cdot E_{i}\right)$. This graph is called the dual intersection graph of the desingularization $\pi$ of $(X, x)$.

A normal surface singularity $(X, x)$ is called a rational singularity if there is a desingularization $\pi: \tilde{X} \rightarrow U$ of $(X, x)$ such that $H^{1}\left(\tilde{X}, \mathcal{O}_{\tilde{X}}\right)=0$. We also say that the local ring $\mathcal{O}_{X, x}$ is rational. This definition is known to be independent of the desingularization $\pi$ (see e.g. [2], Theorem 2.3). From the proof of Proposition 1 in [1], we deduce the following:

Theorem 1.1. Let $(X, x)$ be a rational surface singularity. Let $\rho: X^{\prime} \longrightarrow U$ be a proper map of a normal space $X^{\prime}$ onto a neighbourhood $U$ of $x$ in $X$, where $U-\{x\}$ is non-singular, and which induces an isomorphism between open dense subsets of $X^{\prime}$ and $U$, and is not an isomorphism. Let $D$ be a positive cycle with support in the exceptional divisor $\rho^{-1}(x)$. Then we have $H^{1}\left(|D|, \mathcal{O}_{D}\right)=0$ where $|D|$ is the reduced curve associated with $D$.

Note that, in place of considering a desingularization of $(X, x)$ as in Proposition 1 of [1], we have a modification $\rho: X^{\prime} \rightarrow U$, where $X^{\prime}$ might be singular. As an important consequence, we have:

Corollary 1.2. The irreducible components of the fibre $\rho^{-1}(x)$ are rational nonsingular curves.

Another important result of [1] is (consequence of Theorem 1.1):

Theorem 1.3. If $(X, x)$ is a rational singularity, any desingularization of $(X, x)$ is very good.

Notice that the dual intersection graph of the exceptional divisor of a desingularization of a surface with a rational singularity is a tree.

\section{Rational trees}

Let $\Gamma$ be a graph without loops, with vertices $E_{1}, \cdots, E_{n}$, weighted by pairs $\left(w_{i}, g_{i}\right)$ at each vertex $E_{i},(1 \leq i \leq n)$, where $w_{i}$ is a positive integer called the weight of $E_{i}$, and $g_{i}$ is a non-negative integer, called the genus of $E_{i}$.

With $\Gamma$ we associate a symmetric matrix $\mathcal{M}(\Gamma)=\left(\alpha_{i j}\right)_{1 \leq i, j \leq n}$ in the following way: $\alpha_{i i}=-w_{i}$ and $\alpha_{i j}$ is the number of edges linking the vertices $E_{i}$ and $E_{j}$ whenever $i \neq j$. We call $\mathcal{M}(\Gamma)$ the incidence matrix of $\Gamma$. 
In the free abelian group $\mathcal{G}$ generated by the vertices $E_{i}$ of $\Gamma$, the incidence matrix $\mathcal{M}(\Gamma)$ defines a symmetric bilinear form. We shall denote $(Y \cdot Z)$ the value of this bilinear form on a pair $(Y, Z)$ of elements in $\mathcal{G}$. The elements of $\mathcal{G}$ will be called cycles of the graph $\Gamma$. A positive cycle is a cycle in which all the coefficients are non-negative and at least one is positive. The support of a positive cycle $Y=\sum_{i} m_{i} E_{i}$ is the set of vertices such that $m_{i} \neq 0$.

A weighted graph is called a singular graph if the associated incidence matrix is negative definite.

Theorem 2.1 (see [3], [12]). The dual intersection graph of a desingularization of a normal surface singularity is a singular graph.

By a proof analogous to the one of Zariski (see [20], Theorem 7.1) in the case of curve configurations, we obtain that, for any singular graph $\Gamma$ with vertices $E_{i}$, there are non-zero cycles $Y=\sum_{i=1}^{n} m_{i} E_{i}$ of $\Gamma$ such that $\left(Y \cdot E_{i}\right) \leq 0$ for any $i$, $(1 \leq i \leq n)$; by using the connectivity of the graph, these elements satisfy $m_{i} \geq 1$. As in [11] (see $\S 18)$, let $\mathcal{E}^{+}(\Gamma)$ denote the set of these elements. It is an additive monoïd.

Let $A$ be a set of positive cycles supported on the set of all the vertices of the singular graph $\Gamma$. We define $\inf A$ as $\inf A=Z_{0}=\sum_{i=1}^{n} a_{i} E_{i}$ where

$$
a_{i}=\inf _{Y \in A}\left\{\operatorname{mult}_{Y} E_{i}\right\}
$$

and mult $_{Y} E_{i}$ is the coefficient $m_{i}$ of $E_{i}$ in the positive cycle $Y$. The cycle $Z_{0}$ is a positive cycle since $m_{i} \in \mathbf{N}^{*}$ for any $i$.

Using ([1], [11]), we have:

Theorem 2.2. Let $\Gamma$ be a singular graph. For any subset $A$ of $\mathcal{E}^{+}(\Gamma)$, we have $\inf A \in \mathcal{E}^{+}(\Gamma)$.

Therefore, following [1]:

Definition 2.3. We define $Z_{\Gamma}:=\inf \mathcal{E}^{+}(\Gamma)$ the fundamental cycle of the singular graph $\Gamma$.

By Proposition 4.1 of [9], we find the fundamental cycle of a given graph $\Gamma$ by constructing a sequence of positive cycles in the following way: Put $Z_{1}=\sum_{i=1}^{n} E_{i}$. If $\left(Z_{1} \cdot E_{i}\right) \leq 0$ for all $i$, then $Z_{1}=Z_{\Gamma}$; otherwise, there exists an $E_{i}$, say $E_{i_{1}}$, such that $\left(Z_{1} \cdot E_{i_{1}}\right)>0$, in this case, put $Z_{2}=Z_{1}+E_{i_{1}}$. The term $Z_{j},(j \geq 1)$, of the sequence satisfies, either $\left(Z_{j} \cdot E_{i}\right) \leq 0$ for all $i$, then we put $Z_{\Gamma}=Z_{j}$, or there is an irreducible component $E_{i_{j}}$ such that $\left(Z_{j} \cdot E_{i_{j}}\right)>0$, then we put $Z_{j+1}=Z_{j}+E_{i_{j}}$. Then, the fundamental cycle of $\Gamma$ is the first cycle $Z_{k}$ of this sequence such that $\left(Z_{k} \cdot E_{i}\right) \leq 0$ for all $i$. This construction is called the Laufer algorithm.

Based on Artin's [1] characterization, Spivakovsky ([15], chap. II, def. 1.9), defines: 
Definition 2.4 (Rationality Conditions). A graph $\Gamma$ is called rational if:

(i) it is a tree,

(ii) the set $\mathcal{F}$ of non-zero cycles $D=\sum_{i=1}^{n} k_{i} E_{i}$, where the $k_{i}$ 's are positive integers, such that $\left(D \cdot E_{i}\right) \leq 0$ for all $i=1, \cdots, n$, is not empty and there is $D_{0} \in \mathcal{F}$ such that $D_{0}^{2}<0$,

(iii) The genera $g_{i}$ of all the vertices of $\Gamma$ are trivial,

(iv) Let $\inf \mathcal{F}=Z_{\Gamma}=\sum a_{i} E_{i}$. Then

$$
\frac{1}{2}\left(Z_{\Gamma} \cdot Z_{\Gamma}+\sum_{i=1}^{n} a_{i}\left(w_{i}-2\right)\right)+1=0 .
$$

For a positive cycle $Y=\sum m_{i} E_{i}$, it will be convenient to denote $p(Y):=$ $\frac{1}{2}\left(Y . Y+\sum_{i} m_{i}\left(w_{i}-2\right)\right)+1$ and call it the arithmetic genus of the cycle $Y$. So, by the condition (iv) above, we mean that the arithmetic genus of $Z_{\Gamma}$ is zero. Moreover, the condition (ii) is equivalent to saying that $\Gamma$ is a singular graph and a graph satisfying the conditions (ii) and (iv) is a tree. To prove these facts, we need to relate these graphs to the geometry of singular surfaces (see [1], Proposition 2, or $[18])$.

By plumbing (see [10]), a weighted graph $\Gamma$ defines a (non-unique) complex curve configuration, with smooth components and transverse intersections, embedded in a non-singular complex analytic surface. By a result of Grauert (see [5], p. 367), if a weighted graph $\Gamma$ is singular, there is a normal complex analytic surface singularity and a desingularization of this singularity such that the dual intersection graph of its exceptional divisor is $\Gamma$.

As in [15] (chap. II, Proposition 1.11), we have:

Theorem 2.5. If $\mathcal{R}$ is a rational tree, there is a surface with a rational singularity and a desingularization of this singularity such that the dual intersection graph of its exceptional divisor is $\mathcal{R}$.

Conversely (see also [15]):

Theorem 2.6. If $(X, x)$ is a rational singularity, the dual intersection graph of the exceptional divisor of any desingularization of $(X, x)$ is a rational tree.

\section{Properties of rational trees}

The following proposition is an important consequence of the relation between rational singularities and rational trees:

Proposition 3.1 (see [1]). A weighted tree $\mathcal{R}$ on which the genus of all vertices are 0 , is rational if and only if the arithmetic genus of any positive cycle $Y$ supported on $\mathcal{R}$ is negative, i.e. we have $p(Y) \leq 0$. 
Then we have:

Proposition 3.2. Any subtree of a rational tree is rational.

Proof. Let $\mathcal{R}^{\prime}$ be a subtree of $\mathcal{R}$. The tree $\mathcal{R}^{\prime}$ is singular ([8], Lemma 5.11). Since any positive cycle $Y$ of $\mathcal{R}^{\prime}$ is also a positive cycle of $\mathcal{R}$, by Proposition 3.1, we have $p(Y) \leq 0$. Therefore $\mathcal{R}^{\prime}$ is a rational tree.

We define the valency of the vertex $E_{i}$ in $\mathcal{R}$ to be the number of vertices adjacent to $E_{i}$ in $\mathcal{R}$. We denote it by $v_{\mathcal{R}}\left(E_{i}\right)$. We prove the following property of a rational tree, given by M. Spivakovsky ([15], remark 2.3):

Proposition 3.3. If $\mathcal{R}$ is a rational tree, for any vertex $E_{i}$ of $\mathcal{R}$, we have

$$
w_{i}+1 \geq v_{\mathcal{R}}\left(E_{i}\right) .
$$

Proof. First, assume that $\mathcal{R}$ corresponds to the minimal desingularization $\pi$ : $X \longrightarrow S$ of a rational singularity of $S$ i.e. $w_{i} \geq 2$ for any $E_{i}$. Suppose that in $\mathcal{R}$ there is a vertex $E$ with valency $v_{\mathcal{R}}(E) \geq w_{E}+2$. Consider a subtree $\mathcal{R}^{\prime}$ of $\mathcal{R}$ which contains $E$ and vertices $E_{1}, \ldots E_{v_{\mathcal{R}^{\prime}}(E)}$ adjacent to $E$. Assume $v_{\mathcal{R}^{\prime}}(E)=w_{E}+2$. Since $\mathcal{R}$ is rational, the subtree $\mathcal{R}^{\prime}$ must be rational. We consider the positive cycle defined by:

$$
Z_{\mathcal{R}^{\prime}}=2 E+\sum_{i=1}^{v_{\mathcal{R}^{\prime}}(E)} E_{i} .
$$

This gives $Z_{\mathcal{R}^{\prime}}^{2}=8-\sum_{i=1}^{w_{E}+2} w_{i}$. So, we obtain $p\left(Z_{\mathcal{R}^{\prime}}\right)=1$. This contradicts the fact that $\mathcal{R}^{\prime}$ is rational. Then $w_{E}+1 \geq v_{\mathcal{R}}(E)$.

Now, if $\mathcal{R}$ corresponds to a desingularization of a rational singularity which is not a minimal desingularization, the result is proved by induction on the number of point blow-ups from the minimal desingularization to our desingularization.

Definition 3.4. We call a bad (resp. good) vertex a vertex $E_{i}$ of $\mathcal{R}$ such that $w_{i}+1=v_{\mathcal{R}}\left(E_{i}\right)$ (resp. $w_{i} \geq v_{\mathcal{R}}\left(E_{i}\right)$ ). In particular, we call a very good vertex a vertex $E_{i}$ of $\mathcal{R}$ such that $w_{i}>v_{\mathcal{R}}\left(E_{i}\right)$.

Theorem 3.5. Let $\mathcal{R}$ be a rational tree where the weights are $\geq 2$ and containing two bad vertices and let $C$ be the smallest path (i.e. the geodesic) in the tree $\mathcal{R}$ linking these two bad vertices without containing them. Then, at least one of the vertices of the subtree $C$ is very good.

Proof. Let $E$ and $F$ be the two bad vertices of $\mathcal{R}$. Assume that $C$ is not empty. Let $A_{1}, \cdots, A_{n}$ be the vertices of $C$ such that $\left(A_{i} \cdot A_{j}\right)=1$ for $j=i+1,\left(A_{1} \cdot E\right)=$ $\left(A_{n} \cdot F\right)=1,\left(A_{j} \cdot E\right)=0$ for $j \neq 1$ and $\left(A_{j} \cdot F\right)=0$ for $j \neq n$. Consider the subtree $\mathcal{R}^{\prime}$ of $\mathcal{R}$ which contains $E, F, C$ and the vertices adjacent to the vertices 
$E, F$ and adjacent to $C$. Assume that the vertices of $C$ are all good vertices, but not very good. Since $\mathcal{R}$ is rational, the subtree $\mathcal{R}^{\prime}$ has to be rational. We consider the positive cycle $Z_{\mathcal{R}^{\prime}}$ defined by:

$$
Z_{\mathcal{R}^{\prime}}=\sum_{j=1}^{w_{E}} E_{j}+2 E+\sum_{i=1}^{n} 2 A_{i}+2 F+\sum_{m=1}^{w_{F}} F_{m}+\sum_{i=1}^{k} \sum_{l=1}^{w_{n_{i}}-2} B_{l}^{n_{i}}
$$

where $E_{j}, F_{m}$ et $B_{l}^{n_{i}}$ are the vertices in $\mathcal{R}^{\prime}$ adjacent to $E, F$ and to $A_{n_{i}}$ respectively. We obtain $p\left(Z_{\mathcal{R}^{\prime}}\right)=1$. This contradicts the hypothesis. Therefore the subtree $C$ contains at least one very good vertex.

When $C$ is empty, we have a similar proof. In this case, the positive cycle $Z_{\mathcal{R}^{\prime}}=\sum_{j=1}^{w_{E}} E_{j}+2 E+2 F+\sum_{m=1}^{w_{F}} F_{m}$ of $\mathcal{R}^{\prime}$ gives also $p\left(Z_{\mathcal{R}^{\prime}}\right)=1$, which is again a contradiction.

Definition 3.6. The vertex $E_{i}$ is called a rupture vertex of $\mathcal{R}$ if $v_{\mathcal{R}}\left(E_{i}\right) \geq 3$.

Remark 3.7. Theorem 3.5 implies that a rational tree in which all the weights are equal to 2 has at most one rupture vertex. Of course, this fact is already known, since in this case, the possible trees are $\mathbf{A}_{\mathbf{n}}, \mathbf{D}_{\mathbf{n}}, \mathbf{E}_{\mathbf{6}}, \mathbf{E}_{\mathbf{7}}$ and $\mathbf{E}_{\mathbf{8}}$.

The following result which has been conjectured by M. Spivakovsky gives many rational trees once one of them is known.

Theorem 3.8. Let $\mathcal{R}$ be a rational tree. Let $\mathcal{R}^{\prime}$ be a tree obtained from $\mathcal{R}$ by increasing the weights. Then $\mathcal{R}^{\prime}$ is a rational tree.

Proof. Let $\mathcal{R}$ and $\mathcal{R}^{\prime}$ be trees defined as in the theorem. First we will show that $\mathcal{R}^{\prime}$ is a singular tree: Let us denote by (.) and (.) the bilinear forms defined on the free abelian group generated by the vertices of $\mathcal{R}$ and $\mathcal{R}^{\prime}$ respectively. Let $Y=\sum_{i=1}^{n} b_{i} E_{i}$ be a positive cycle such that $\left(Y \cdot E_{i}\right) \leq 0$ for any $i$. Since $\left(E_{i} \cdot E_{i}\right)^{\prime} \leq\left(E_{i} \cdot E_{i}\right)$ for any $i$, we have $\left(Y \cdot E_{i}\right)^{\prime} \leq\left(Y \cdot E_{i}\right) \leq 0$ for any $i$. By the same method, we obtain $(Y \cdot Y)^{\prime 2} \leq(Y \cdot Y)^{2}<0$. By (ii) of Definition 2.4, $\mathcal{R}^{\prime}$ is a singular tree.

Now let us denote $p(Y)$ and $p^{\prime}(Y)$ the arithmetic genus of a positive cycle $Y$ defined by taking the bilinear forms in $\mathcal{R}$ and $\mathcal{R}^{\prime}$ respectively. Let $D=\sum_{i=1}^{n} a_{i}^{\prime} E_{i}$ be a positive cycle of $\mathcal{R}^{\prime}$. Since $D$ can be considered as a positive cycle with support on $\mathcal{R}$, Proposition 3.1 implies that $p(D) \leq 0$. Hence it will be sufficient to show that $p^{\prime}(D) \leq p(D)$.

We prove this last assertion by induction on the number of vertices where the two weighted trees $\mathcal{R}$ and $\mathcal{R}^{\prime}$ differ. Therefore, it is sufficient to prove the assertion when they differ only at one vertex, say $E_{1}$. The condition (iv) of Definition 2.4 gives:

$$
p^{\prime}(D)-p(D)=\frac{a_{1}^{\prime 2}\left(E_{1} \cdot E_{1}\right)^{\prime}+a_{1}^{\prime} w_{1}^{\prime}}{2}-\frac{a_{1}^{\prime 2}\left(E_{1} \cdot E_{1}\right)+a_{1}^{\prime} w_{1}}{2} .
$$


Since $\left(E_{1} \cdot E_{1}\right)^{\prime}=-w_{1}^{\prime}$ and, by hypothesis, $w_{1}^{\prime}=w_{1}+k, k \in \mathbf{N}^{*}$, we obtain

$$
p^{\prime}(D)-p(D)=\frac{k a_{1}^{\prime}\left(-a_{1}^{\prime}+1\right)}{2} .
$$

Since $a_{1}^{\prime} \geq 1$, we have $p^{\prime}(D)-p(D) \leq 0$.

The preceding proof also shows that:

Proposition 3.9. With the notation of the theorem above, if the weight of a vertex in $\mathcal{R}^{\prime}$ is strictly greater than its weight in $\mathcal{R}$, the multiplicity of that vertex in the fundamental cycle of $\mathcal{R}^{\prime}$ equals 1 .

We do not have a complete classification of rational trees. However, there are strong restrictions for a tree to be rational, as stated in the following theorem:

Theorem 3.10. Let $\mathcal{R}$ be a rational tree. The vertices of a subtree $\mathcal{R}^{\prime}$ of $\mathcal{R}$ whose valency in $\mathcal{R}$ is different from its valency in $\mathcal{R}^{\prime}$, have multiplicity 1 in the fundamental cycle of $\mathcal{R}^{\prime}$.

Proof. To prove the theorem, we make use of the geometrical meaning of a rational tree.

Let $(X, x)$ be a surface with a rational singularity for which $\mathcal{R}$ is the dual intersection tree associated to the exceptional divisor of a desingularization $\pi: \tilde{X} \rightarrow$ $(X, x)$.

Let $F$ be a vertex of $\mathcal{R}^{\prime}$ whose valency in $\mathcal{R}^{\prime}$ is not the same as in $\mathcal{R}$, and let $E$ be a vertex in $\mathcal{R}-\mathcal{R}^{\prime}$ which is adjacent to $F$.

Theorem 2.5 says that, by contracting all the components of the exceptional divisor of $\pi$ which correspond to the vertices of $\mathcal{R}^{\prime}$, we obtain a normal surface $S^{\prime}$ having a rational singularity and bimeromorphic morphisms $\kappa: \tilde{X} \rightarrow S^{\prime}$ and $\rho: S^{\prime} \rightarrow(X, x)$ such that $\pi=\rho \circ \kappa$. Denote the singularity of $S^{\prime}$ by $\xi_{1}$. Since the morphism $\rho$ is bimeromorphic and $(X, x)$ is a rational singularity, Corollary 1.2 shows that the components of $\rho^{-1}(x)$ are non-singular rational curves. In particular $\kappa(E)$, which is a component of $\rho^{-1}(x)$, is a non-singular rational curve.

A result of Gonzalez-Sprinberg and Lejeune-Jalabert in [4] implies that, since the curve $\kappa(E)$ is non-singular, the strict transform of $\kappa(E)$ by $\kappa$ intersects the exceptional divisor of $\kappa$ transversally at a component which has coefficient 1 in the fundamental cycle in $\kappa^{-1}\left(\xi_{1}\right)$ of the singularity $\xi_{1}$. Since $\mathcal{R}^{\prime}$ is a rational tree, $\left(S^{\prime}, \xi_{1}\right)$ is a rational singularity and the maximal divisor in $\kappa^{-1}\left(\xi_{1}\right)$ of the singularity $\xi_{1}$ coincides with the fundamental cycle of $\kappa$. This cycle corresponds to the fundamental cycle of $\mathcal{R}^{\prime}$. Therefore, the coefficient of $F$ in this fundamental cycle is 1 .

Corollary 3.11. The rational tree $\mathbf{E}_{\mathbf{8}}$ cannot be a subtree strictly contained in a rational tree. 
This comes from the fact that the coefficients of all the vertices of $\mathbf{E}_{\mathbf{8}}$ in its fundamental cycle are $\geq 2$.

\section{Glueing rational trees}

Let $\Gamma_{1}$ and $\Gamma_{2}$ be two weighted trees. The weighted tree $\Gamma$ obtained by attaching a vertex of $\Gamma_{1}$ and a vertex of $\Gamma_{2}$ by an edge is called the glueing tree of $\Gamma_{1}$ and $\Gamma_{2}$ at these vertices.

Through this section, we will denote by $\mathcal{R}_{1}$ and $\mathcal{R}_{2}$ the rational trees with vertices $E_{1}, \cdots, E_{n}$ and $F_{1}, \cdots, F_{m}$ respectively.

Another corollary of Theorem 3.10 is:

Corollary 4.1. If the glueing tree of $\mathcal{R}_{1}$ and $\mathcal{R}_{2}$ at $E_{1}$ and $F_{1}$ is rational, the coefficient of $E_{1}$ (resp. $F_{1}$ ) in the fundamental cycle of $\mathcal{R}_{1}$ (resp. $\mathcal{R}_{2}$ ) is 1 .

Proof. In the glueing tree, the weights of the vertices don't change, but the valencies of $E_{1}$ and $F_{1}$ change. Theorem 3.10 gives the result.

Remark 4.2. We may always consider a rational tree to be made of vertices of weight $\geq 3$ and rational subtrees of type $\mathbf{A}_{\mathbf{n}}, \mathbf{D}_{\mathbf{n}}, \mathbf{E}_{\mathbf{6}}, \mathbf{E}_{\mathbf{7}}$ or $\mathbf{E}_{\mathbf{8}}$ (see prop. 3.2). The vertices of these subtrees which are linked to a vertex of weight $\geq 3$ have coefficient 1 in the fundamental cycle of the corresponding subtree. We saw that $\mathbf{E}_{\mathbf{8}}$ cannot be the strict subtree of a rational tree. In the case of $\mathbf{E}_{\mathbf{6}}$, we cannot glue any tree to any vertex of $\mathbf{E}_{\mathbf{6}}$, except at the ends of the long tails, since the coefficients of other vertices are $\geq 2$ in the fundamental cycle of $\mathbf{E}_{\mathbf{6}}$. Similarly, to obtain rational trees by glueing $\mathbf{E}_{\mathbf{7}}$, only one end vertex is available and, for $\mathbf{D}_{\mathbf{n}}$, only the ends are available. However, there are rational trees obtained by glueing $\mathbf{A}_{\mathbf{n}}$ at any of its points.

The following theorem shows that the glueing of rational trees gives a rational tree only under some important necessary conditions:

Theorem 4.3. Let $Z_{1}$ and $Z_{2}$ be the fundamental cycles of $\mathcal{R}_{1}$ and $\mathcal{R}_{2}$ respectively. Assume that the glueing tree $\mathcal{R}$ of $\mathcal{R}_{1}$ and $\mathcal{R}_{2}$ at the vertices $E_{1}$ and $F_{1}$ is rational. Then either $\left(Z_{1} \cdot E_{1}\right)<0$ or $\left(Z_{2} \cdot F_{1}\right)<0$.

Before giving a proof of this theorem, it will be useful to introduce the following definitions (compare with Definition III.3.1 in [15]):

Definition 4.4. Let $\Gamma$ be a singular graph. A vertex $E$ of $\Gamma$ is called non-Tjurina for an element $Y$ of $\mathcal{E}^{+}(\Gamma)$ if it satisfies $(Y . E)<0$.

A connected component of the difference $\Gamma-\{$ non-Tjurina vertices for $Y\}$ is called a Tjurina component for the element $Y$ in $\mathcal{E}^{+}(\Gamma)$. 
Let $\mathcal{R}$ be a rational tree. A vertex $E$ is non-Tjurina for the fundamental cycle $Z_{\mathcal{R}}$ if and only if it corresponds to the strict transform of a component of the tangent cone of a rational singularity whose dual intersection tree of a desingularization is $\mathcal{R}$. Moreover, a result of Tjurina ([17]) implies that a Tjurina component of the fundamental cycle $Z_{\mathcal{R}}$ is the dual intersection tree of a desingularization of one of the rational singularities which appear after the point blowing-up of the rational singularity of the surface whose dual intersection graph of a desingularization is $\mathcal{R}$.

Now, let us introduce the desingularization depth of a vertex in a rational tree: Let $E$ be a vertex in $\mathcal{R}$. Then, $E$ is either non-Tjurina for $Z_{\mathcal{R}}$, or is contained in a Tjurina component $\Delta_{1}$ for $Z_{\mathcal{R}}$. In the first case, we say that the desingularization depth of the vertex $E$ is zero; in the second case, $E$ is either non-Tjurina for the fundamental cycle $Z_{\Delta_{1}}$ of $\Delta_{1}$, or is contained in a Tjurina component $\Delta_{2}$ for $Z_{\Delta_{1}}$. By induction, we define the desingularization sequence of the vertex $E$ in $\mathcal{R}$ as the sequence $\Delta_{0}=\mathcal{R}, \Delta_{1}, \ldots, \Delta_{p}$ of subtrees of $\mathcal{R}$ such that, for all $i,(1 \leq i \leq p), E$ is a vertex of $\Delta_{i}, \Delta_{i}$ is the Tjurina component of $\Delta_{i-1}$ for the fundamental cycle $Z_{\Delta_{i-1}}$ and $E$ is a non-Tjurina in $\Delta_{p}$ for the fundamental cycle $Z_{\Delta_{p}}$. In this case, $p$ is called the desingularization depth of $E$. We will also call the degree of the vertex $E$ the number $-\left(Z_{\mathcal{R}} . E\right)$.

Proof of Theorem 4.3. The theorem states that, if the glueing tree $\mathcal{R}$ is rational then, either $E_{1}$ is non-Tjurina for $Z_{1}$, or $F_{1}$ is non-Tjurina for $Z_{2}$.

Now assume that the glueing tree $\mathcal{R}$ is rational. Let $\Delta_{0}, \Delta_{1}, \ldots, \Delta_{p}$ be the desingularization sequence of the vertex $E_{1}$ in $\mathcal{R}_{1}$ and $\mathcal{D}_{0}, \mathcal{D}_{1}, \ldots, \mathcal{D}_{q}$ be the desingularization sequence of the vertex $F_{1}$ in $\mathcal{R}_{2}$. Then

$$
U:=\sum_{i=0}^{p} Z_{\Delta_{i}}+\sum_{j=0}^{q} Z_{\mathcal{D}_{j}}
$$

is a positive cycle of $\mathcal{R}$. The Proposition 3.1 shows that the arithmetic genus $p(U)$ of $U$ is $\leq 0$.

Furthermore, by Theorem 3.10, the coefficient of $E_{1}$ (resp. $F_{1}$ ) in $Z_{1}$ (resp. $Z_{2}$ ) is one. The following lemma shows that the coefficients of $E_{1}$ (resp. $F_{1}$ ) in $Z_{\Delta_{i}}$ (resp. $Z_{\mathcal{D}_{j}}$ ) are also 1 , for any $i, 0 \leq i \leq p$ (resp. for any $j, 0 \leq j \leq q$ ).

Lemma 4.5. Let $Z_{\mathcal{R}}=\sum_{i=1}^{n} a_{i} E_{i}$ and $Z_{\mathcal{R}^{\prime}}=\sum_{i \in A^{\prime}} a_{i}^{\prime} E_{i}, A^{\prime} \subset\{1, \cdots, n\}$, be the fundamental cycles of $\mathcal{R}$ and of a subtree $\mathcal{R}^{\prime}$ of $\mathcal{R}$ respectively. Then we have $a_{i}^{\prime} \leq a_{i}$ for $i \in A^{\prime}$.

We shall give below a proof of this lemma. Let us continue the proof of Theorem 4.3. We have

$$
p(U)=\sum_{i=0}^{p} p\left(Z_{\Delta_{i}}\right)+\sum_{j=0}^{q} p\left(Z_{\mathcal{D}_{j}}\right)-(p+1+q+1)+1+\sum_{0 \leq i \leq p, 0 \leq j \leq q}\left(Z_{\Delta_{i}} \cdot Z_{\mathcal{D}_{j}}\right) .
$$


Since, for $k<\ell, \Delta_{\ell}$ (resp. $\mathcal{D}_{\ell}$ ) is contained in a Tjurina component of $\Delta_{k}$ (resp. $\left.\mathcal{D}_{k}\right)$, we have $\left(Z_{\Delta_{k}} \cdot Z_{\Delta_{\ell}}\right)=0$ (resp. $\left(Z_{\mathcal{D}_{k}} \cdot Z_{\mathcal{D}_{\ell}}\right)=0$ ) for all $k \neq \ell$. On the other hand, since the coefficients of $E_{1}$ (resp. $F_{1}$ ) in the fundamental cycles $Z_{\Delta_{i}}$ (resp. $Z_{\mathcal{D}_{j}}$ ) are 1 , we have

$$
\left(Z_{\Delta_{i}} \cdot Z_{\mathcal{D}_{j}}\right)=1
$$

for any $i, j,(0 \leq i \leq p),(0 \leq j \leq q)$.

Now, any subtree of a rational tree being rational, we have $p\left(Z_{\Delta_{i}}\right)=0$ for any $i,(0 \leq i \leq p)$, and $p\left(Z_{\mathcal{D}_{j}}\right)=0$ for any $j,(0 \leq j \leq q)$. Therefore

$$
p(U)=(p+1)(q+1)-(p+1+q+1)+1 .
$$

Since $p(U) \leq 0$, we have $p q \leq 0$, which implies either $p=0$ or $q=0$. This proves Theorem 4.3.

It remains to prove Lemma 4.5.

Proof of Lemma 4.5. Let $Z_{r}$ be the positive cycle defined as the restriction of $Z$ to $A^{\prime}$, denoted by $\left.Z\right|_{A^{\prime}}=Z_{r}=\sum_{i \in A^{\prime}} a_{i} E_{i}$. For $i \in A^{\prime}$, we have

$$
\left(Z_{r} \cdot E_{i}\right)=a_{i}\left(E_{i} \cdot E_{i}\right)+\sum_{j \neq i, j \in A^{\prime}} a_{j}\left(E_{j} \cdot E_{i}\right) .
$$

Furthermore, the fact that $\left(Z \cdot E_{i}\right) \leq 0$ for all $i$ gives

$$
-a_{i}\left(E_{i} \cdot E_{i}\right) \geq \sum_{j \neq i} a_{j}\left(E_{j} \cdot E_{i}\right) .
$$

Since $a_{j} \geq 0$ and $\left(E_{j} \cdot E_{i}\right) \geq 0$ for $i \neq j$, we have

$$
\sum_{j \neq i} a_{j}\left(E_{j} \cdot E_{i}\right) \geq \sum_{j \neq i, j \in A^{\prime}} a_{j}\left(E_{j} \cdot E_{i}\right) .
$$

Then we obtain

$$
-a_{i}\left(E_{i} \cdot E_{i}\right) \geq \sum_{j \neq i, j \in A^{\prime}} a_{j}\left(E_{j} \cdot E_{i}\right) .
$$

So we have $\left(Z_{r} \cdot E_{i}\right) \leq 0$ for all $i \in A^{\prime}$. Let $Z^{\prime}$ be the fundamental cycle of $\mathcal{R}^{\prime}$. The fact $Z^{\prime} \leq Z_{r}$ gives $a_{i}^{\prime} \leq a_{i}$ for any $i \in A^{\prime}$.

Theorem 4.3 gives the following result:

Theorem 4.6. Let $\mathcal{R}_{1}$ and $\mathcal{R}_{2}$ be rational trees and $Z_{1}$ and $Z_{2}$ be their fundamental cycles respectively. Assume that the weights of $\mathcal{R}_{2}$ are $\geq 2,\left(Z_{1} \cdot E_{1}\right)<0$ and the degree of $E_{1}, d_{1}=-\left(Z_{1} \cdot E_{1}\right)$, in $\mathcal{R}_{1}$ is strictly greater than the desingularization depth of $F_{1}$ in $\mathcal{R}_{2}$, then the glueing tree $\mathcal{R}$ of $\mathcal{R}_{1}$ and $\mathcal{R}_{2}$ at $E_{1}$ and $F_{1}$ is rational.

Proof. Using the same notation to that of the proof of Theorem 4.3, we shall prove 
that the positive cycle

$$
U:=Z_{1}+\sum_{j=0}^{q} Z_{\mathcal{D}_{j}}
$$

of the glueing tree $\mathcal{R}$ is in fact the fundamental cycle of $\mathcal{R}$, so $p(U)=0$. First, let us check that $(U \cdot E) \leq 0$ for any vertex $E$ of $\mathcal{R}$. For this purpose, we need the following lemma that we shall prove later:

Lemma 4.7. Let $E$ be a vertex of $\mathcal{R}$ with coefficient 1 in the fundamental cycle $Z_{\mathcal{R}}$. Let $p$ be the desingularization depth of $E$ and $\Delta_{0}=\mathcal{R}, \Delta_{1}, \ldots, \Delta_{p}$ be its desingularization sequence. For any $i, 1 \leq i \leq p$, the cycle $\sum_{0}^{i} Z_{\Delta_{k}}$ belongs to $\mathcal{E}^{+}(\mathcal{R})$ and it is the smallest cycle in $\mathcal{E}^{+}(\mathcal{R})$ greater than $\sum_{0}^{i-1} Z_{\Delta_{k}}+E$.

We have $\left(\sum_{j=0}^{q} Z_{\mathcal{D}_{j}} \cdot F_{i}\right) \leq 0$ for all vertices $F_{i}$ of $\mathcal{R}_{2}$. Since $\left(Z_{1} \cdot F_{i}\right)=0$ for $i \neq 1$, we have $\left(U \cdot F_{i}\right) \leq 0$ for all $F_{i}$ with $i=2, \cdots m$. Similarly, $\left(U \cdot E_{i}\right) \leq 0$ for all vertices $E_{i}$ of $\mathcal{R}_{1}$ with $i=2, \cdots n$. Hence it remains to estimate $\left(U \cdot E_{1}\right)$ and $\left(U \cdot F_{1}\right)$. We have

$$
\left(U \cdot E_{1}\right)=\left(Z_{1} \cdot E_{1}\right)+\left(\sum_{j=0}^{q} Z_{\mathcal{D}_{j}} \cdot E_{1}\right) .
$$

However $-\left(Z_{1} \cdot E_{1}\right)$ is the degree $d_{1}$ of $E_{1}$ in $\mathcal{R}_{1}$ and, by Corollary 4.1 and Lemma $4.5,\left(Z_{\mathcal{D}_{j}} \cdot E_{1}\right)=1$ for any $j,(0 \leq j \leq q)$, so that $\left(\sum_{0}^{q} Z_{\mathcal{D}_{j}} \cdot E_{1}\right)=q+1$. Since $d_{1}>q,\left(U \cdot E_{1}\right) \leq 0$. Now, consider

$$
\left(U \cdot F_{1}\right)=\left(Z_{1} \cdot F_{1}\right)+\left(\sum_{0}^{q} Z_{\mathcal{D}_{j}} \cdot F_{1}\right) .
$$

Since $\left(Z_{1} \cdot F_{1}\right)=1$ and $\left(\sum_{0}^{q} Z_{\mathcal{D}_{j}} \cdot F_{1}\right)=\left(Z_{\mathcal{D}_{q}} \cdot F_{1}\right) \leq-1$, we obtain $\left(U \cdot F_{1}\right) \leq 0$. Then $U$ is an element of $\mathcal{E}^{+}(\mathcal{R})$. Moreover, we obtain $(U \cdot U)<0$ because we have either $\left(Z_{\mathcal{D}_{q}} \cdot F_{1}\right)<-1$ or $\left(Z_{\mathcal{D}_{q}} \cdot F_{1}\right)=-1$. The first case gives $\left(U \cdot F_{1}\right)<0$. In the second case, since by assumption the weights of all the vertices of $\mathcal{D}_{q}$ are $\geq 2$, there is necessarily another non-Tjurina vertex $F \neq F_{1}$ of $\mathcal{D}_{q}$ such that $\left(Z_{\mathcal{D}_{q}} \cdot F\right)<0$, so $(U \cdot U)<0$. Therefore $\mathcal{R}$ is a singular tree.

Let us prove that $U$ is the fundamental cycle $Z_{\mathcal{R}}$ of $\mathcal{R}$. In the case the desingularization depth of $F_{1}$ is $0, U=Z_{1}+Z_{2}$. We just proved that $U \in \mathcal{E}^{+}(\mathcal{R})$, so $U \geq Z_{\mathcal{R}}$. However Lemma 4.5 tells us that the restriction of $Z_{\mathcal{R}}$ to $\mathcal{R}_{1}$ and $\mathcal{R}_{2}$ are in $\mathcal{E}^{+}\left(\mathcal{R}_{1}\right)$ and $\mathcal{E}^{+}\left(\mathcal{R}_{2}\right), Z_{\mathcal{R}} \leq Z_{1}+Z_{2}$ which gives

$$
Z_{\mathcal{R}}=Z_{1}+Z_{2} \text {. }
$$

When the desingularization depth of $F_{1}$ is $\geq 1$, since

$$
U \geq Z_{\mathcal{R}}
$$

Lemma 4.5 and the Laufer algorithm say that the restriction of the fundamental cycle $Z_{\mathcal{R}}$ to $\mathcal{R}_{2}$ belongs to $\mathcal{E}^{+}\left(\mathcal{R}_{2}\right)$ and is greater than $Z_{2}+F_{1}=Z_{\mathcal{D}_{0}}+F_{1}$. By 
Lemma 4.7 , it is also greater than $Z_{\mathcal{D}_{0}}+Z_{\mathcal{D}_{1}}$. By induction, we show that the restriction of $Z_{\mathcal{R}}$ to $\mathcal{R}_{2}$ is greater than $\sum_{0}^{q} Z_{\mathcal{D}_{j}}$. Therefore we have $Z_{\mathcal{R}} \geq U=$ $\sum_{j=0}^{q} Z_{\mathcal{D}_{j}}+Z_{1}$, and so $Z_{\mathcal{R}}=U$ as expected.

Finally, we show that $p(U)=0$. We have

$$
p(U)=p\left(Z_{1}\right)+\sum_{0}^{q} p\left(Z_{\mathcal{D}_{j}}\right)+\sum_{0}^{q}\left(Z_{1} \cdot Z_{\mathcal{D}_{j}}\right)-q-1 .
$$

Since $p\left(Z_{1}\right)=0$ and $p\left(Z_{\mathcal{D}_{j}}\right)=0$ for any $j,(0 \leq j \leq q)$, we have

$$
p(U)=\sum_{0}^{q}\left(Z_{1} \cdot Z_{\mathcal{D}_{j}}\right)-q-1 .
$$

By Corollary 4.1 and Lemma 4.5 , the coefficients of $E_{1}$ in $Z_{1}$ and $F_{1}$ in $Z_{\mathcal{D}_{j}}$ for any $j,(0 \leq j \leq q)$, are equal to 1 . Then $\left(Z_{1} \cdot Z_{\mathcal{D}_{j}}\right)=1$ and $p(U)=0$. This completes the proof of the Theorem 4.6.

We have also a similar result:

Theorem 4.8. Let $\mathcal{R}_{1}$ and $\mathcal{R}_{2}$ be rational trees and $Z_{1}$ and $Z_{2}$ be their fundamental cycles respectively. Assume that $\left(Z_{1} \cdot E_{1}\right)<0$ and the degree of $E_{1}$, $d_{1}=-\left(Z_{1} \cdot E_{1}\right)$, in $\mathcal{R}_{1}$ is strictly greater than the desingularization depth of $F_{1}$ in $\mathcal{R}_{2}$ plus one, then the glueing tree $\mathcal{R}$ of $\mathcal{R}_{1}$ and $\mathcal{R}_{2}$ at $E_{1}$ and $F_{1}$ is rational.

The proof of this Theorem is similar to the one of Theorem 4.6. In this case we have $\left(U \cdot E_{1}\right)<0$, because $d_{1}>q+1$. This is enough to get $(U \cdot U)<0$ and obtain that $\mathcal{R}$ is a singular tree.

Now let us prove Lemma 4.7 .

Proof of Lemma 4.7. We shall give a proof by induction on $i$. For $i=0$, it is the definition of the fundamental cycle of $\Delta_{0}:=\mathcal{R}$. For $i=1$, it is the result of the proposition of $\S 14$ in [14] (see p. 165 or [19]). Now, let $i \geq 2$. We assume that the Lemma is true for $\ell,(0 \leq \ell \leq i-1)$.

Denote $U_{i}=\sum_{k=0}^{i} Z_{\Delta_{k}}$. Let $F$ be a vertex of $\mathcal{R}$. Assume that $\left(U_{i} \cdot F\right)>0$. Consider

$$
\left(\sum_{k=0}^{i} Z_{\Delta_{k}} \cdot F\right)=\left(\sum_{k=0}^{i-1} Z_{\Delta_{k}} \cdot F\right)+\left(Z_{\Delta_{i}} \cdot F\right) .
$$

Since, by induction, we have $\left(\sum_{k=0}^{i-1} Z_{\Delta_{k}} \cdot F\right) \leq 0$, necessarily $\left(Z_{\Delta_{i}} \cdot F\right)>0$. Hence $F \notin \Delta_{i}$, but it is linked to $\Delta_{i}$ at a vertex $F^{\prime}$ of $\Delta_{i}$. Since $\Delta_{i}$ is a Tjurina component of $\Delta_{i-1}$, the point $F^{\prime}$ is also in $\Delta_{i-1}$.

If $F$ belongs to $\Delta_{i-1}$, then $\left(\left(Z_{\Delta_{i-1}}+Z_{\Delta_{i}}\right) \cdot F\right) \leq 0$ by applying the result of the Proposition of $\S 14$ in [14]. Since $\left(\sum_{k=0}^{i-2} Z_{\Delta_{k}} \cdot F\right) \leq 0$ by the induction hypothesis, we obtain $\left(\sum_{k=0}^{i} Z_{\Delta_{k}} \cdot F\right) \leq 0$, which contradicts the assumption $\left(U_{i} \cdot F\right)>0$ above. So, the vertex $F$ cannot belong to $\Delta_{i-1}$. 
The restriction $Z_{i-1}:=Z_{\mathcal{R}} \mid \Delta_{i-1}$ of the fundamental cycle $Z_{\mathcal{R}}$ to $\Delta_{i-1}$ belongs to $\mathcal{E}^{+}\left(\Delta_{i-1}\right)$ (see the proof of 4.5 ). So $Z_{i-1} \geq Z_{\Delta_{i-1}}$. However, these cycles are different, since $\left(Z_{i-1} \cdot F^{\prime}\right)<0$, because $F^{\prime}$ is linked to $F$ in $\mathcal{R}$, and $\left(Z_{\Delta_{i-1}} \cdot F^{\prime}\right)=0$, since $F^{\prime}$ is in a Tjurina component of $\Delta_{i-1}$. Let $Z_{i-1}=Z_{\Delta_{i-1}}+Y$. We prove that $Y \geq F^{\prime}$. We have $\left(Z_{i-1} \cdot F^{\prime}\right)<0$, which yields $\left(Y \cdot F^{\prime}\right)<0$. Therefore $F^{\prime}$ is in the support of $Y$. This shows $Z_{i-1} \geq Z_{\Delta_{i-1}}+F^{\prime}$. The Proposition of $\S 14$ in [14] then implies that

$$
Z_{i-1} \geq Z_{\Delta_{i-1}}+Z_{\Delta_{i}}
$$

which implies that the coefficient of $E$ in the fundamental cycle $Z_{\mathbb{R}}$ is $\geq 2$. This, again, contradicts the hypothesis on the coefficient of $E$ in $Z_{\mathbb{R}}$. Therefore, for any vertex $F$ of $\mathcal{R}$, we must have $\left(U_{i} . F\right) \leq 0$.

It remains to prove that, for any $i,(1 \leq i \leq p)$, the cycle $\sum_{0}^{i} Z_{\Delta_{k}}$ is the smallest cycle in $\mathcal{E}^{+}(\mathcal{R})$ amongst the cycles greater than $\sum_{0}^{i-1} Z_{\Delta_{k}}+E$. Let

$$
Y=\sum_{0}^{i-1} Z_{\Delta_{k}}+E+T
$$

be a positive cycle in $\mathcal{E}^{+}(\mathcal{R})$. For any $F$ in $\Delta_{i}$ we have $(Y . F) \leq 0$. By Lemma 4.5 the restriction $T^{\prime}$ of the cycle $E+T$ to the subtree $\Delta_{i}$ is in $\mathcal{E}^{+}\left(\Delta_{i}\right)$, so that $T^{\prime} \geq Z_{\Delta_{i}}$, which implies $Y \geq \sum_{0}^{i} Z_{\Delta_{k}}$. Since we proved that $\sum_{0}^{i} Z_{\Delta_{k}}$ is in $\mathcal{E}^{+}(\mathcal{R})$, it is the smallest cycle in $\mathcal{E}^{+}(\mathcal{R})$ which is greater than $\sum_{0}^{i-1} Z_{\Delta_{k}}+E$. This ends the proof of Lemma 4.7.

\section{Complexity of rational trees}

We say that a rational tree $\mathcal{R}$ has multiplicity $m$ if its fundamental cycle satisfies $\left(Z_{\mathcal{R}} \cdot Z_{\mathcal{R}}\right)=-m$. According to [1] (see Theorem 4 ), the number $-\left(Z_{\mathcal{R}} \cdot Z_{\mathcal{R}}\right)$ is the multiplicity of a rational singularity having $\mathcal{R}$ as dual intersection graph of a desingularization.

In this paragraph, we want to prove that the complexity of a rational tree of given multiplicity is bounded. In the cases of multiplicity 2,3 and 4 , it has been observed (see [3], [1] and [16]) that there are a finite number of types of rational trees with weights $\geq 2$. It is therefore of interest to have a better understanding of this result. The first problem is to give a proper definition of the complexity. Since rupture vertices of the dual intersection graph of the minimal good desingularization measure the local topological complexity of the link of a complex normal surface singularity (see [13]), it seems natural to define the complexity of a rational tree whose vertices have weights $\geq 2$ to be the number of rupture vertices. This idea is enhanced by the following result: 
Theorem 5.1. Let $\mathcal{R}$ be a rational tree of multiplicity $m$ in which all vertices have weight $\geq 2$. Then the number of rupture vertices of $\mathcal{R}$ is bounded by $m-2$ if $m \geq 3$.

Remark 5.2. The multiplicity of a rational tree with weights $\geq 2$ is equal to 2 if and only if all its vertices have weight 2 (see [3]). As pointed out above, these rational trees are $\mathbf{A}_{\mathbf{n}}, \mathbf{D}_{\mathbf{n}}, \mathbf{E}_{\mathbf{6}}, \mathbf{E}_{\mathbf{7}}$ and $\mathbf{E}_{\mathbf{8}}$, and the number of rupture vertices in any of these trees is equal to 0 or 1 (see [1]). Of course, in the Theorem we could replace the bound by $m-1$ to include the cases of multiplicity 2 , but in the following example, we give an infinite class of rational trees with arbitrary multiplicity $m \geq 3$ for which the bound $m-2$ is reached.

Example 5.3. Let us consider the following tree $\mathcal{R}$ with vertices $E_{1}, \cdots, E_{n}$ :

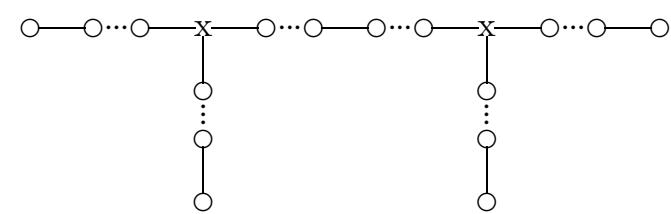

where "o" and "x" denote the vertices with weights 2 and 3 respectively. The fundamental cycle of this tree is $Z_{\mathcal{R}}=\sum_{i=1}^{n} E_{i}$. If the number of rupture vertices of weight 3 is $k$, then $Z_{\mathcal{R}}^{2}=-(k+2)$. Since $p\left(Z_{\mathcal{R}}\right)=0, \mathcal{R}$ is indeed a rational tree with multiplicity $(k+2)$ and $k$ rupture vertices.

Lemma 5.4. Assume that $\mathcal{R}$ is a rational tree of multiplicity $m$ in which all vertices have weight $\geq 2$. Assume that $\mathcal{R}$ contains a unique vertex with weight $\geq 3$. Then the number of rupture vertices of $\mathcal{R}$ is $\leq m-2$.

Proof. Denote by $F$ the vertex which has weight $\geq 3$ and by $E_{1}, \cdots, E_{n}$ the vertices of weight 2 in $\mathcal{R}$. Let $Z_{\mathcal{R}}=a_{F} F+\sum_{i=1}^{n} a_{i} E_{i}$ be the fundamental cycle of $\mathcal{R}$. Since $Z_{\mathcal{R}}^{2}=-m$ and $p\left(Z_{\mathcal{R}}\right)=0$, we need to show that:

$$
m-2=a_{F}\left(w_{F}-2\right) \geq s,
$$

where $s$ is the number of rupture vertices of $\mathcal{R}$. In what follows, the case $s=1$, for which the inequality is trivial, is excluded.

Let us denote $\mathcal{R}_{1}, \cdots, \mathcal{R}_{p}$ the maximal subtrees of $\mathcal{R}-\{F\}$. Obviously, all the vertices of $\mathcal{R}_{j},(j=1, \cdots, p)$, are of weight 2 , and each $\mathcal{R}_{j}$ is of type $\mathbf{A}_{\mathbf{n}}, \mathbf{D}_{\mathbf{n}}, \mathbf{E}_{\mathbf{6}}$ or $\mathbf{E}_{\mathbf{7}}$, since $\mathbf{E}_{\mathbf{8}}$ has been excluded by Corollary 3.11. Denote by $E_{j}$ the vertex of $\mathcal{R}_{j}$ adjacent to $F(j=1, \ldots, p)$ in $\mathcal{R}$. This gives $v_{\mathcal{R}}(F)=p$. Then the rupture vertices of $\mathcal{R}$ are maybe $F$ itself, the possible rupture vertices of the subtrees $\mathcal{R}_{j}$, $(1 \leq j \leq p)$, and the rupture vertices obtained by glueing $F$ and all the $\mathcal{R}_{j}$ 's. We know, by remark 4.2 , that the glueing of $F$ and one $\mathcal{R}_{j}$ can give a rupture vertex only in the case where $F$ is attached to an interior vertex of $\mathcal{R}_{j}$ which is of type $\mathbf{A}_{\mathbf{n}}$. 
Let us denote by $\alpha$ the number of subtrees among $\mathcal{R}_{1}, \cdots, \mathcal{R}_{p}$ which have a rupture vertex or which gives a rupture vertex at $E_{j}$ when attached to $F$, and by $\beta$ the number of subtrees among $\mathcal{R}_{1}, \cdots, \mathcal{R}_{p}$ which are of type $\mathbf{A}_{\mathbf{n}}$ and which are attached to $F$ by an extremity vertex. Then the number of rupture vertices of $\mathcal{R}$ is equal to $\alpha$ if $p \leq 2$, and it is equal to $\alpha+1$ if $p \geq 3$ (i.e. accordingly $F$ becomes a rupture vertex in $\mathcal{R}$ upon its glueing to the $\mathcal{R}_{j}$ 's).

Let $a_{F}$ and $a_{1}, \cdots, a_{p}$ be the coefficients of $F$ and $E_{1}, \cdots, E_{p}$ in $Z_{\mathcal{R}}$ respectively. The fact that $\left(Z_{\mathcal{R}} \cdot F\right) \leq 0$ gives:

$$
w_{F} a_{F} \geq \sum_{j=1}^{p} a_{j} .
$$

Lemma 5.5. If a subtree $\mathcal{R}_{j}$ has a rupture vertex or if it contributes to a rupture vertex at $E_{j}$ when it is glued to $F$, then $a_{j} \geq 2$.

Proof. It is obvious that the coefficient of a bad vertex in the fundamental cycle of a rational tree is $\geq 2$. Since the rupture vertex of $\mathcal{R}_{j}$ is a bad vertex and there are only vertices with weight 2 on the geodesic from the rupture vertex of $\mathcal{R}_{j}$ to $F$, we can easily see that the vertex of $\mathcal{R}_{j}$ adjacent to $F$ has the coefficient $\geq 2$ in the fundamental cycle of $\mathcal{R}$.

We deduce that $a_{F} w_{F} \geq 2 \alpha+\beta$, so $\frac{1}{2} a_{F} w_{F} \geq \alpha$. Lemma 5.4 will be proved upon showing that:

$$
a_{F}\left(w_{F}-2\right)>\frac{1}{2} a_{F} w_{F}
$$

as this implies that $a_{F}\left(w_{F}-2\right) \geq \alpha+1$. In fact, the preceding inequality is equivalent to $\left(w_{F}-2\right)>\frac{1}{2} w_{F}$ or $w_{F}>4$. So Lemma 5.4 is true for $\mathbf{w}_{\mathbf{F}} \geq \mathbf{5}$.

Remark 5.6. If $\beta>0$ then the Lemma 5.4 is true for $w_{F} \geq 4$.

Using essentially Theorem 3.5 and Lemma 5.5, we may now finish the proof of Lemma 5.4:

For $\quad \mathbf{w}_{\mathbf{F}}=3$ : By Proposition 3.3, we have $p \leq 4$. Theorem 3.5 gives that $s \leq 2$. The only case to be treated is when $s=2$, which happens if $p=2$ or $p=3$. When $p=2, \mathcal{R}$ is constructed by two subtrees $\mathcal{R}_{j}(j=1,2)$ attached to $F$ and each of these subtrees has at most one rupture vertex in $\mathcal{R}$, so $s=\alpha$. If $s=\alpha=2$, since $a_{F} w_{F}=3 a_{F} \geq 2 \alpha+\beta \geq 2 \alpha=4$, we have $a_{F} \geq 2$, so that $a_{F}\left(w_{F}-2\right)=a_{F} \geq 2=s$.

When $p=3, \mathcal{R}$ is constructed by three subtrees $\mathcal{R}_{j}$ attached to $F$, since $F$ becomes a good vertex without being very good, at most one of these subtrees has a rupture vertex in $\mathcal{R}$ and $s=\alpha+1$. If $s=2$, we have $\alpha=1$ and $\beta=2$, so $a_{F} w_{F}=3 a_{F} \geq 2 \alpha+\beta=4$ and $a_{F} \geq 2$. This also implies

$$
a_{F}\left(w_{F}-2\right)=a_{F} \geq 2=s .
$$


For $\mathbf{w}_{\mathbf{F}}=4$ : We have $p \leq 5$. The inequality (1) is obvious when $p \leq 2$ and $4 \leq p \leq 5$ for which we have $s \leq 2$. When $p=3$, we obtain $s \leq 4$. So we have two cases to prove: If $s=3$ (resp. $s=4$ ), $F$ is a rupture vertex and there exist two (resp. three) subtrees $\mathcal{R}_{j}$ which have a rupture vertex in $\mathcal{R}$. Then

$$
a_{F} w_{F}=4 a_{F} \geq 2 \alpha+\beta=5 \text { (resp. } a_{F} w_{F}=4 a_{F} \geq 2 \alpha+\beta=6 \text { ). }
$$

In both cases, we have $a_{F} \geq 2$. Thus we obtain $a_{F}\left(w_{F}-2\right)=2 a_{F} \geq 4 \geq s$.

Proof of Theorem 5.1. We shall make use of Lemma 5.4 and the fact that $\mathcal{R}$ is the union of trees with only one vertex of weight $\geq 3$.

Let us denote by $F_{1}, \cdots, F_{k}$ the vertices with weights $\geq 3$ and by $E_{1}, \cdots, E_{n}$ the vertices with weights 2 in $\mathcal{R}$. Let $Z_{\mathcal{R}}=\sum_{i=1}^{k} a_{i} F_{i}+\sum_{n=1}^{n} b_{j} E_{j}$ be the fundamental cycle of $\mathcal{R}$. Observe that $m \geq 3$ implies $k \geq 1$. So we must show:

$$
m-2=\sum_{i=1}^{k} a_{i}\left(w_{i}-2\right) \geq s
$$

where $s$ is the number of rupture vertices in $\mathcal{R}$.

For each vertex $F_{i},(1 \leq i \leq k)$, we consider the maximal subtree $\mathcal{B}_{i}$ of $\mathcal{R}$ which contains $F_{i}$ and all subtrees $\mathcal{R}_{j}, j \in J_{i}$, of $\mathcal{R}-\left\{F_{1}, \cdots, F_{k}\right\}$ which are adjacent to $F_{i}$. We know that $\mathcal{B}_{i}$ is a rational tree. Moreover, $\mathcal{B}_{i}$ has the form of the rational tree given in Lemma 5.4 , so each $\mathcal{B}_{i}$ satisfies $a_{i}^{\prime}\left(w_{i}-2\right) \geq s_{i}$ where $Z_{i}=a_{i}^{\prime} F_{i}+\sum_{E_{l} \in \mathcal{R}_{i}} a_{l}^{\prime} E_{l}$ is the fundamental cycle of $\mathcal{B}_{i}$ and $s_{i}$ is the number of rupture vertices of $\mathcal{B}_{i}$. By Lemma 4.5 , we have

$$
m-2=\sum_{i=1}^{k} a_{i}\left(w_{i}-2\right) \geq \sum_{i=1}^{k} a_{i}^{\prime}\left(w_{i}-2\right) \geq \sum_{i=1}^{k} s_{i} .
$$

We cannot assert that $\sum_{i=1}^{k} s_{i} \geq s$, since it is possible to create some rupture vertices which are not rupture vertices in the $\mathcal{B}_{i}$ 's.

Lemma 5.7. A rupture vertex in $\mathcal{R}$ which is not a rupture vertex in any $\mathcal{B}_{i}$ is of the following types:

(a) it is the vertex $F_{i}$ of valency $\leq 2$ in $\mathcal{B}_{i}$.

(b) it is a vertex $E_{j}$ which is the extremity of one of the subtrees $\mathcal{R}_{j}$ of $\mathcal{B}_{i}$ which is of type $\mathbf{A}_{\mathbf{n}}$.

Proof. This follows from the construction of $\mathcal{B}_{i}$ and from Theorem 3.5. In fact, if a rupture vertex in $\mathcal{R}$, which is not a rupture vertex in any $\mathcal{B}_{i}$, has weight 2 , it is necessarily a bad vertex in $\mathcal{R}$. By Theorem 3.5 , the subtree $\mathcal{R}_{j}$ of $\mathcal{B}_{i}$ which contains that vertex must be of type $\mathbf{A}_{\mathbf{n}}$. Moreover, it is an extremity of this subtree of type $\mathbf{A}_{\mathbf{n}}$ because if it was not, it would already be a rupture vertex in $\mathcal{B}_{i}$ 's which contain it.

Thus, we shall consider vertices of a subtree $\mathcal{B}_{i}$ which are rupture vertices in $\mathcal{R}$ without being rupture vertices in the subtree $\mathcal{B}_{i}$ in the following cases: 
(A) the vertex $F_{i}$ of $\mathcal{B}_{i}$,

(B) vertices of weight 2 which are extremities of subtrees $\mathcal{R}_{j}$ of type $\mathbf{A}_{\mathbf{n}}$,

(C) vertices of the preceding two types.

5.8. To find the rupture vertices of $\mathcal{R}$ which are not rupture vertices in $\mathcal{B}_{i}$, it is sufficient to consider the attachment to $\mathcal{B}_{i}$ of the vertices in $\mathcal{R}-\mathcal{B}_{i}$ which are adjacent to $\mathcal{B}_{i}$. By construction of $\mathcal{B}_{i}$, these vertices, which are adjacent to $\mathcal{B}_{i}$ in $\mathcal{R}$, have weights $\geq 3$. Let us denote by $\mathcal{B}_{i}^{\prime}$ a minimal subtree of $\mathcal{R}$ which contains $\mathcal{B}_{i}$ and in which the vertices which become rupture vertices in $\mathcal{R}$ without being rupture vertices in $\mathcal{B}_{i}$ are rupture vertices. Let $s_{i}^{\prime}$ be the number of rupture vertices in $\mathcal{B}_{i}^{\prime}$. If $\mathcal{B}_{i}$ does not contain rupture vertices of $\mathcal{R}$ which are not rupture vertices in $\mathcal{B}_{i}$, we set $\mathcal{B}_{i}^{\prime}=\mathcal{B}_{i}$ and $s_{i}^{\prime}=s_{i}$.

Let $a_{F_{i}}^{\prime \prime}$ be the coefficient of $F_{i}$ in the fundamental cycle of $\mathcal{B}_{i}^{\prime}$. By Lemma 4.5 we have $a_{F_{i}} \geq a_{F_{i}}^{\prime \prime}$. Since $\sum_{i=1}^{k} s_{i}^{\prime} \geq s$, Theorem 5.1 will be proved if we show, in the three cases above, that:

$$
a_{F_{i}}^{\prime \prime}\left(w_{F_{i}}-2\right) \geq s_{i}^{\prime} .
$$

(A) Let us consider a subtree $\mathcal{B}_{i}$ of $\mathcal{R}$ which contains a rupture vertex of weight $\geq 3$ of $\mathcal{R}$ which is not a rupture vertex in that $\mathcal{B}_{i}$.

Denote by $\mathcal{B}$ such a subtree $\mathcal{B}_{i}$ and by $F$ its unique vertex with weight $\geq$ 3. Since $F$ is not a rupture vertex in $\mathcal{B}, \mathcal{B}-\{F\}$ has at most two connected components. By hypothesis, we have $s^{\prime}=s+1$ where $s^{\prime}$ is the number of rupture vertices of a minimal subtree $\mathcal{B}^{\prime}$ of $\mathcal{R}$ which contains $\mathcal{B}$ and in which $F$ is a rupture vertex. So there are three cases to be proved depending on the valency of $F$ in $\mathcal{B}$ :

(1) If $v_{\mathcal{B}}(F)=0$, we have $\mathcal{B}=\{F\}$. A minimal subtree $\mathcal{B}^{\prime}$ is obtained by glueing three vertices with weight $\geq 3$ of $\mathcal{R}$ to $E$. The inequality (4) is obvious for $w_{F} \geq 3$.

(2) If $v_{\mathcal{B}}(F)=1$, the rational tree $\mathcal{B}$ consists of the vertex $F$ attached to a tree of type $\mathbf{A}_{\mathbf{n}}, \mathbf{D}_{\mathbf{n}}, \mathbf{E}_{\mathbf{6}}$ or $\mathbf{E}_{\mathbf{7}}$ attached to the vertex $F$. A minimal subtree $\mathcal{B}^{\prime}$ of $\mathcal{R}$ is obtained by glueing two vertices of $\mathcal{R}$ with weight $\geq 3$ to the vertex $F$ of $\mathcal{B}$. We have $s^{\prime} \leq 2$. Since the inequality (4) is obvious for $w_{F} \geq 4$, the only case to consider is when $w_{F}=3$ and $s^{\prime}=2$. This gives, by Lemma 5.5 and inequality (2), $3 a_{F}^{\prime \prime} \geq 4$. Thus $a_{F}^{\prime \prime} \geq 2$, and we have inequality (4).

(3) If $v_{\mathcal{B}}(F)=2, \mathcal{B}$ is obtained by glueing two subtrees of type $\mathbf{A}_{\mathbf{n}}, \mathbf{D}_{\mathbf{n}}, \mathbf{E}_{\mathbf{6}}$ or $\mathbf{E}_{\mathbf{7}}$ to the vertex $F$. A minimal subtree $\mathcal{B}^{\prime}$ contains $\mathcal{B}$ and a vertex with weight $\geq 3$ of $\mathcal{R}$ attached to $F$. Since $s^{\prime} \leq 3$, our inequality is obvious when $w_{F} \geq 5$. Now, we have $s^{\prime} \leq 2$ (resp. $\left.s^{\prime} \leq 3\right)$ if $w_{F}=3$ (resp. $\left.w_{F}=4\right)$. When $s^{\prime}=2$ (resp. $s^{\prime}=3$ ), by Lemma 5.5 and inequality $(2)$, we obtain $3 a_{F}^{\prime \prime} \geq 4$ (resp. $4 a_{F}^{\prime \prime} \geq 5$ ). This gives $a_{F}^{\prime \prime} \geq 2$, and so we also have inequality (4) for $w_{F}=3$ and 4 .

(See lemme 7.1 and lemme 7.4 in [18] for all possible rational trees of the type given in cases (2) and (3) respectively.) 
(B) Let us consider a subtree $\mathcal{B}_{i}$ of $\mathcal{R}$ which contains rupture vertices of $\mathcal{R}$ which are not the rupture vertices in $\mathcal{B}_{i}$ and assume that these vertices have weight 2:

Denote by $\mathcal{B}$ such a subtree $\mathcal{B}_{i}$. As in the proof of Lemma 5.4, among the maximal subtrees of vertices of weight 2 of $\mathcal{B}$, we have:

(i) the $\alpha$ maximal subtrees with vertices of weight 2 which contain a rupture vertex of $\mathcal{B}$,

(ii) the $\gamma$ maximal subtrees with vertices of weight 2 which contain a rupture vertex of $\mathcal{R}$ which is not a rupture vertex in $\mathcal{B}$. We saw that these subtrees are of type $\mathbf{A}_{\mathbf{n}}$ and they are attached to the vertex with weight $\geq 3$ by one extremity.

(iii) the $\beta$ maximal subtrees with vertices of weight 2 of $\mathcal{B}$ which contain no rupture vertex of $\mathcal{R}$.

By Lemma 5.5 and inequality (2), we have $a_{F} w_{F} \geq 2 \alpha+2 \gamma+\beta$ where $F$ is the vertex with weight $\geq 3$ in $\mathcal{B}$. As in the case $(\mathbf{A})$, let $\mathcal{B}^{\prime}$ be a minimal subtree of $\mathcal{R}$ which contains $\mathcal{B}$ and in which the vertices of weight 2 of $\mathcal{B}$ which are rupture vertices in $\mathcal{R}$ without being rupture vertices in $\mathcal{B}$, are also rupture vertices. Since $s=\alpha$ or $\alpha+1$, we have $s^{\prime}=\alpha+\gamma$ or $s^{\prime}=\alpha+\gamma+1$. If $\mathbf{w}_{\mathbf{F}} \geq \mathbf{5}$, Theorem 5.1 is proved in the case $(\mathbf{B})$, because we have $a_{F}\left(w_{F}-2\right)>\frac{1}{2} a_{F} w_{F} \geq \alpha+\gamma$.

It only remains to prove the result for the cases $w_{F}=3$ and $w_{F}=4$. First, notice that we have $\gamma \geq 1$.

$\mathbf{w}_{\mathbf{F}}=\mathbf{3}$ : Theorem 3.5 shows that the valency of $F$ is necessarily $\leq 3$ and, if the valency is 3 , the only possibility is $s^{\prime}=1$. In this case,

$$
a_{F}\left(w_{F}-2\right)=a_{F} \geq 1=s^{\prime} .
$$

If the valency of $F$ is $\leq 2, s^{\prime} \leq 2$. The case $s^{\prime}=1$ has just been considered. When $s^{\prime}=2$, we have $\alpha+\gamma=2$, and $a_{F} w_{F}=3 a_{E} \geq 2 \alpha+2 \gamma+\beta \geq 4$, which implies $a_{F} \geq 2$, so $a_{F}\left(w_{F}-2\right) \geq 2=s^{\prime}$.

$\boldsymbol{w}_{\boldsymbol{F}}=4$ : By Theorem 3.5, the valency of $F$ is $\leq 4$ and, if it is $4, s^{\prime}=1$, in which case, the inequality $a_{F}\left(w_{F}-2\right) \geq s^{\prime}$ is true.

We may assume that the valency of $F$ is $\leq 3$. Then $s^{\prime} \leq 4$. The case $s^{\prime}=1$ has already been treated. In the same way, for $s^{\prime}=2$, our inequality holds. Thus, we may suppose that the valency of $E$ is 3 . Then, for $\alpha+\gamma=2$ and $\beta=1$ (resp. $\alpha+\gamma=3$ and $\beta=0$ ), we have $s^{\prime}=3$ (resp. $s^{\prime}=4$ ). We have

$$
\begin{gathered}
a_{F} w_{F}=4 a_{F} \geq 2(\alpha+\gamma)+\beta \geq 5 \\
\text { (resp. } \left.a_{F} w_{F}=4 a_{F} \geq 2(\alpha+\gamma)+\beta \geq 6\right),
\end{gathered}
$$

which implies in both cases $a_{F}=2$ and, so $a_{F}\left(w_{F}-2\right)=2 a_{F} \geq 4 \geq s^{\prime}$.

(C) Consider a subtree $\mathcal{B}_{i}$ of $\mathcal{R}$ containing rupture vertices with weights 2 and $\geq 3$ of $\mathcal{R}$ which are not rupture vertices in that $\mathcal{B}_{i}$.

Denote by $\mathcal{B}$ such a subtree $\mathcal{B}_{i}$ of $\mathcal{R}$ and by $F$ the unique vertex in $\mathcal{B}_{i}$ of weight $\geq 3$. Since $F$ is not a rupture vertex in $\mathcal{B}$ but becomes a rupture vertex in $\mathcal{R}$, we have necessarily that $v_{\mathcal{B}}(F) \leq 2$ (see the case $(\mathbf{A})$ above). This implies that $\mathcal{B}$ 
contains at most two rupture vertices with weights 2 of $\mathcal{R}$ which are not rupture vertices in $\mathcal{B}$. Then we consider a minimal subtree $\mathcal{B}^{\prime}$ of $\mathcal{R}$ which contains $\mathcal{B}$ and in which $F$ and the vertices of $\mathcal{B}$, which are rupture vertices in $\mathcal{R}$ without being the rupture vertices in $\mathcal{B}$ are rupture vertices. Then, we only have the cases $s=0$ or $s=1$, since $s=2$ would imply $\gamma=0$, contradicting our hypothesis (C). So $s^{\prime}=2$ or $s^{\prime}=3$. Therefore we have $a_{F}^{\prime \prime}\left(w_{F}-2\right) \geq s^{\prime}$ for $w_{F} \geq 5$. Again, it remains to prove this inequality for $w_{F}=3$ and $w_{F}=4$.

$\boldsymbol{w}=3$ Let $s^{\prime}=2$, then $\gamma=1, \alpha=0$ and $\beta=1$. Then $F$ being a rupture point in $\mathcal{B}^{\prime}$, there are another vertex of weight $\geq 3$ adjacent to $F$ in $\mathcal{B}^{\prime}$, we have $a_{F}^{\prime \prime} w_{F}=3 a_{F}^{\prime \prime} \geq 2(\alpha+\gamma)+\beta+1$. So $a_{F}^{\prime \prime} \geq 2$, which gives the desired inequality $a_{F}^{\prime \prime}\left(w_{F}-2\right) \geq s^{\prime}$.

If $s^{\prime}=3$, then $\alpha+\gamma=2$. In $\mathcal{B}^{\prime}$ there is a subtree $\mathcal{B}_{1}$ containing $F$ and $\mathcal{B}$, where $F$ is not a rupture point, but where a vertex of weight 2 is a rupture point in $\mathcal{B}_{1}$ and not in $\mathcal{B}$. In $\mathcal{B}_{1}$, we have

$$
a_{F}^{1} w_{F} \geq 2(\alpha+\gamma)+\beta=4
$$

where $a_{F}^{1}$ is the coefficient of $F$ in the fundamental cycle of $\mathcal{B}_{1}$. Therefore $a_{F}^{1} \geq 2$. Since $\mathcal{B}^{\prime}$ is obtained from $\mathcal{B}_{1}$ by glueing points of weight $\geq 3$, it cannot be rational, because Corollary 4.1 implies that $a_{F}^{1}=1$.

$\boldsymbol{w}=4$ The inequality is obvious for $s^{\prime}=2$. Suppose that $s^{\prime}=3$. Then, $\alpha+\gamma=2$ and $a_{F}^{\prime \prime} w_{F}=4 a_{F} \geq 2(\alpha+\gamma)+\beta+1$, which gives $a_{F}^{\prime \prime} \geq 2$ and the desired inequality.

This ends the proof of Theorem 5.1.

\section{Some classes of Rational Trees}

There exist interesting classes of rational trees having some nice properties. In this section we define these classes and we discuss a few of their properties.

(1) A rational tree is called minimal rational tree if all its weights are $\geq 2$ and the coefficients of all vertices in the fundamental cycle are 1 (see [15], p. 425). Kollár in ([7], 4.4.10) has shown that a normal surface singularity is minimal if and only if the dual intersection graph associated with the exceptional divisor of the minimal desingularization of the singularity is rational and minimal. Another simple characterization of rational minimal trees, due to Spivakovsky is:

Proposition 6.1. ([15], remark 2.3) A tree $\mathcal{R}$ with weights $\geq 2$ is minimal rational if and only if, for any vertex $E_{i},(1 \leq i \leq n)$, of $\mathcal{R}$, we have $w_{i} \geq v_{\mathcal{R}}(i)$.

Proof. The first implication follows from the fact that we have $\left(Z \cdot E_{i}\right) \leq 0$ for any $i,(1 \leq i \leq n)$. If $\mathcal{R}$ is a tree such that we have $w_{i} \geq v_{\mathcal{R}}(i)$ for any vertex $E_{i}$ in $\mathcal{R}$, then by the Laufer algorithm, we have $Z=\sum_{i=1}^{n} E_{i}$, and $Z^{2}<0$ because at points of valency 1 we have $\left(Z \cdot E_{i}\right)<0$. Since we have $n-1=\frac{1}{2} \sum v_{\mathcal{R}}\left(E_{i}\right)$, we 
obtain

$$
p(Z)=\frac{\sum_{i=1}^{n}\left(-w_{i}+v_{\mathcal{R}}\left(E_{i}\right)\right)+\sum_{i=1}^{n}\left(w_{i}-2\right)}{2}+1=0 .
$$

It is obvious that any subtree of a minimal rational tree is a minimal rational tree. Notice that there are no bad vertices in a minimal rational tree. Moreover:

Proposition 6.2. Let $\mathcal{R}_{1}$ and $\mathcal{R}_{2}$ be two rational minimal trees and let $E_{1}$ (resp. $F_{1}$ ) be a vertex of $\mathcal{R}_{1}$ (resp. $\mathcal{R}_{2}$ ) such that $w_{E_{1}}>v_{\mathcal{R}_{1}}\left(E_{1}\right)$ (resp. $w_{F_{1}}>v_{\mathcal{R}_{2}}\left(F_{1}\right)$ ). Then the glueing tree of $\mathcal{R}_{1}$ and $\mathcal{R}_{2}$ at $E_{1}$ and $F_{1}$ is a rational minimal tree.

Proof. Let $Z_{1}=\sum_{i=1}^{s} E_{i}$ and $Z_{2}=\sum_{j=1}^{t} F_{j}$ be the fundamental cycles of $\mathcal{R}_{1}$ and $\mathcal{R}_{2}$ respectively. Let $E_{1}$ and $F_{1}$ be defined as in the Proposition. Let $\mathcal{R}$ denote the glueing tree at $E_{1}$ and $F_{1}$. It is easy to show, by the Laufer algorithm, that the fundamental cycle of $\mathcal{R}$ exists and that it is exactly $Z=Z_{1}+Z_{2}$. So we have $Z^{2}<0$ and

$$
p(Z)=\frac{\sum_{i=1}^{s} v_{\mathcal{R}_{1}}\left(E_{i}\right)+\sum_{j=1}^{t} v_{\mathcal{R}_{2}}\left(F_{j}\right)+2-2 s-2 t}{2}+1 .
$$

This implies that $p(Z)=0$ and the Proposition as well.

(2) A non-singular tree is the dual intersection tree of an embedded desingularization of a complex plane curve germ. An interesting characterization of a non-singular tree is given by Artin:

Proposition 6.3 (see [1], Theorem 4). A weighted tree is non-singular if and only if it is rational and the self-intersection of its fundamental cycle equals -1 .

However, a subtree of a non-singular tree is not non-singular in general.

(3) Following Spivakovsky (see [15] Definition 1.9), a weighted tree is called sandwich if it is the subtree of a non-singular tree. Since a non-singular tree is rational, any sandwich tree is a rational tree. An interesting characterization of sandwich trees, due to Spivakovsky is:

Proposition 6.4 (see [15], p. 420). A weighted tree is sandwich if and only if, by attaching a finite number of vertices of weight 1 , it becomes a non-singular tree.

This result leads us immediately to:

Proposition 6.5. Let $\mathcal{R}$ be a sandwich tree. Let $\mathcal{R}^{\prime}$ be a tree obtained from $\mathcal{R}$ by increasing the weights. Then $\mathcal{R}^{\prime}$ is a sandwich tree.

Proof. Let $\mathcal{A}$ be a non-singular tree which contains $\mathcal{R}$ as subtree. Let $E$ be a vertex of $\mathcal{A}$ of weight $w$ which belongs to $\mathcal{R}$. Consider the tree $\Delta_{1}$ obtained from 
$\mathcal{A}$ by attaching a vertex $E_{1}$ of weight 1 to $E$. It is easy to see that $\Delta_{1}$ is the dual tree of the exceptional divisor of the embedded desingularization of a plane complex curve. In fact, let $\mathbf{E}$ be the component of the exceptional divisor of an embedded desingularization of a plane complex curve associated to $\mathcal{A}$. Then, by blowing-up a general point of $\mathbf{E}$, we obtain another exceptional divisor of an embedded desingularization of a plane complex curve whose dual graph is precisely $\Delta_{1}$. Proceeding by induction on the difference between the sums of weights of $\mathcal{R}^{\prime}$ and $\mathcal{R}$, we prove our theorem.

\section{References}

[1] M. Artin, On isolated rational singularities of surfaces, Amer. J. Math. 88 (1966), 129-136.

[2] K. Behnke and O. Riemenschneider, Quotient surface singularities and their deformations, Singularity Theory, 1-55.

[3] P. Du Val, On isolated singularities which do not affect the conditions of adjunction, Part I, Proc. Cambridge Phil. Soc. 30 (1934), 453-465.

[4] G. Gonzalez-Sprinberg and M. Lejeune-Jalabert, Courbes lisses sur les singularités de surfaces, C. R. Acad. Sci. Paris Sér. I Math. 318, no. 7 (1994), 653-656.

[5] H. Grauert, Über Modifikationen und exzeptionnelle analytische Mengen, Math. Ann. 146 (1962), 331-368.

[6] R. Hartshorne, Algebraic Geometry, Springer-Verlag, 1984.

[7] J. Kollár, Towards moduli of singular varieties, Compositio Math. 56 (1985), 369-398.

[8] H. Laufer, Normal two-dimensional singularities, Ann. of Math. Studies, 71, Princeton Univ. Press, 1971.

[9] H. Laufer, On rational singularities, Amer. J. Math. 94 (1972), 597-608.

[10] H. Laufer, Taut two-dimensional singularities, Math. Ann. 205 (1973), 131-164.

[11] J. Lipman, Rational singularities, with applications to algebraic surfaces and unique factorization, Publ. Math. IHES 36 (1969), 195-279.

[12] D. Mumford, The topology of normal singularities of an algebraic surface, Publ. Math. IHES 9 (1961), 5-22.

[13] W. Neumann, A calculus for plumbing applied to the topology of complex surface singularities and degenerating complex curves, Trans. of the Amer. Math. Soc. 2682 (1981), 299-344.

[14] H. Pinkham, Singularités rationnelles de surfaces, Séminaire sur les singularités des surfaces, Springer-Verlag, 777, 1980.

[15] M. Spivakovsky, Sandwich singularities and desingularisation of surfaces by normalized Nash transformations, Ann. Math. 131 (1990), 411-491.

[16] J. Stevens, Partial resolutions of rational quadruple points, Int. J. Math. 2 no. 2 (1991), 205-221.

[17] G. Tjurina, Absolute isolatedness of rational singularities and rational triple points, Fonc. Anal. Appl. 2 (1968), 324-332.

[18] M. Tosun, Thèse de doctorat, Université de Provence, 1998.

[19] M. Tosun, Tyurina components and rational cycles for rational singularities, Turkish J. Math. 23, no. 3 (1999), 361-374.

[20] O. Zariski, The theorem of Riemann-Roch for high multiples of an effective divisor on a algebraic surface, Ann. Math. 76 (1962), 560-615. 
Lê Dũng Tráng

Université de Provence

C.M.I.

39 rue F. Joliot-Curie

13453 Marseille Cedex 13

France

e-mail: ledt@gyptis.univ-mrs.fr
Meral Tosun

Instituto de Matematicas U.N.A.M.

Apdo Postal \#273-3

Adm. de Correos \#3

C.P. 62251

Cuernavaca, Morelos

Mexico

e-mail: mtosun@matcuer.unam.mx

(Received: January 22, 2003)

(17) To access this journal online:

(40) http://www.birkhauser.ch 